\title{
Predictors for upstaging of ductal carcinoma in situ (DCIS) to invasive carcinoma in non-mass-type DCIS
}

\author{
GOSHI ODA $^{1}$, TSUYOSHI NAKAGAWA ${ }^{1}$, AYUMI OGAWA ${ }^{1}$, YUICHI KUMAKI ${ }^{1}$, \\ TOKUKO HOSOYA ${ }^{1}$, HITOSHI SUGIMOTO ${ }^{1}$, TOSHIYUKI ISHIBA ${ }^{1}$, MORI MIO ${ }^{2}$, \\ TOMOYUKI FUJIOKA ${ }^{2}$, KAZUNORI KUBOTA ${ }^{2}$, IICHIROH ONISHI ${ }^{3}$ and HIROYUKI UETAKE ${ }^{1}$ \\ Departments of ${ }^{1}$ Surgical Specialties, ${ }^{2}$ Radiology and ${ }^{3}$ Pathology, Tokyo Medical and Dental University, \\ Graduate School of Medicine and Dentistry, Bunkyo-ku, Tokyo 113-8519, Japan
}

Received June 12, 2019; Accepted March 26, 2020

DOI: $10.3892 / \mathrm{mco} .2020 .2036$

\begin{abstract}
Preoperatively diagnosed ductal carcinoma in situ (DCIS) is sometimes upstaged to invasive cancer by postoperative pathological examination. Various preoperative factors associated with upstaging to invasive cancer have been reported; however, this subject remains to be clarified. DCIS takes various forms on imaging, but many cases show non-mass-type lesions. In non-mass-type DCIS, recognizing the presence of invasion is difficult. To investigate predictors associated with upstaging to invasive cancer more precisely, we examined only non-mass-type DCIS. The present study retrospectively analyzed 101 patients diagnosed with non-mass-type DCIS preoperatively on breast biopsy at our institution between 2007 and 2017. Data were analyzed using Fisher's exact probability test and two-sample t-tests. Multivariate analysis was performed using logistic regression. The results showed that 27 patients (27\%) were finally diagnosed with invasive cancer. Univariate analysis revealed abnormal result of palpation on breast examination $(\mathrm{P}=0.05)$, comedo necrosis $(\mathrm{P}=0.05)$, and HER2 status $(\mathrm{P}=0.02)$ as significant predictors. Multivariate analysis revealed an abnormal result of palpation as an independent predictor of invasive cancer underestimation (odds ratio 4.76; confidence interval 1.44-15.7; $\mathrm{P}=0.01$ ). In conclusion, preoperatively diagnosed non-mass-type DCIS represented an underestimation in approximately $27 \%$ of cases. In particular, the presence of a clinically abnormal palpation increases the chance of upstaging to invasive cancer.
\end{abstract}

Correspondence to: Dr Goshi Oda, Department of Surgical Specialties, Tokyo Medical and Dental University, Graduate School of Medicine and Dentistry, 1-5-45 Yushima, Bunkyo-ku, Tokyo 113-8519, Japan

E-mail: odasrg2@tmd.ac.jp

Abbreviations: DCIS, ductal carcinoma in situ; $\mathrm{CNB}$, core-needle biopsy; VAB, vacuum-assisted biopsy; MRI, magnetic resonance imaging; US, ultrasound; Gd-DTPA, gadobenate dimeglumine; MMG, mammography; ST-VAB, stereotactic VAB; ER, estrogen receptor; PgR, progesterone receptor; IHC, immunohistochemistry; FAD, focal asymmetric density

Key words: breast cancer, DCIS, non-mass-type DCIS

\section{Introduction}

Ductal carcinoma in situ (DCIS) is a non-invasive malignant breast disease described as a precursor lesion to invasive breast cancer. With the progress of diagnostic breast imaging, opportunities to diagnose DCIS are on the increase.

If breast cancer is suspected, core-needle biopsy (CNB) or vacuum-assisted biopsy (VAB) is performed. Those percutaneous breast biopsies are important procedures for decreasing the number of excisional biopsies. However, diagnosis from such a biopsy cannot provide definitive diagnosis. For example, a preoperative diagnosis of DCIS is sometimes upstaged to invasive ductal carcinoma (IDC). In the literature, the underestimation rate has been reported as $15-40 \%$ (1-4).

Upstaging to IDC is critical for both patients and surgeons. Surgeons should consider the possibility of underestimation, such as whether to include sentinel lymph node biopsy (SLNB) in a subsequent definitive surgical procedure. Some predictors of underestimation have been reported. Brennan et al reported palpability, nuclear grade, tumor size, and other factors (2). However, reliable predictors have not been clearly identified.

DCIS takes various forms, including solid mass lesion, intracystic lesion, calcification alone, and distortion. An abnormal result from palpation is sometimes a predictor of upstaging to invasive disease (5). However, for lesions showing an intracystic component, the underestimation ratio may not necessarily be high even if the results of palpation are abnormal. Investigating mass-type DCIS or non-mass-type DCIS (Fig. 1) separately is thus important.

This case focused on predictors for underestimation of non-mass-type lesions. Currently, no reports from this point of view have been published. This is thus, to the best of our knowledge, the first report to investigate underestimation, defined as biopsy diagnosis of DCIS with attention only on non-mass-type lesions.

\section{Patients and methods}

Patients. The present study was conducted with approval from the institutional review board of Tokyo Medical and Dental University (IRB-approved number is M2000-831, 2000) and with informed consent of the patient. Data were retrieved from 
our database for 150 consecutive women with an initial CNB or VAB diagnosis of DCIS, and who underwent surgical resection at our institution between September, 2007 and August, 2017. Only 101 cases in which masses could not be confirmed from magnetic resonance imaging (MRI) and ultrasound (US) were examined (Fig. 1).

Non-mass-type lesions were defined from US showing a non-mass lesion (e.g., duct ectasia, intraductal calcification, architectural distortion, clustered microcysts, hypoechoic area) and MRI showing non-mass enhancement (homogeneous, heterogeneous, clumped, or clustered ring). A lack of abnormal findings on US and/or MRI were also considered acceptable.

Imaging evaluation. Mammography examination (craniocaudal and mediolateral oblique views) was performed using a Lorad Selenia mammograph (Hologic). An EUB-7500 scanner with a EUP-L54MA 9.75-MHz linear probe (Hitachi Medical Systems) or Aplio XG scanner with a PLT-805AT 8.0-MHz linear probe (Toshiba Medical Systems) was used for US examinations. MRI examination was performed with a 1.5-T system (Magnetom Vision; Siemens) and a 3.0-T system (Signa HDxt; General Electric Medical Systems) using a breast coil in the prone position. To evaluate the results of MRI, the early phase of a contrast enhancement study within 1 and 2 min after intravenous bolus injection of gadobenate dimeglumine (Gd-DTPA) $(0.2 \mathrm{ml} / \mathrm{kg})$ was obtained. A unilateral coronal T1-weighted sequence [repetition time $(\mathrm{TR})=170 \mathrm{msec}$; echo time $(\mathrm{TE})=4.7 \mathrm{msec}$; flip angle $=40^{\circ} ; 4-\mathrm{mm}$ thick section, $256 \times 256$ matrix; field of view $=210 \mathrm{~mm}$ ] using the 1.5-T system and bilateral axial fat-suppressed T1-weighted sequence $\left(\mathrm{TR}=6.5 \mathrm{msec} ; \mathrm{TE}=2.4 \mathrm{msec}\right.$; flip angle $=10^{\circ}$; section thickness $=2 \mathrm{~mm}$; matrix $=512 \times 512$; field of view $=$ $360 \mathrm{~mm}$ ) using the 3.0-T system were employed.

Examinations were performed by one of three radiologists with $>5$ years of experience in breast imaging. The radiologists had knowledge of the clinical indications for examination and interpreted lesions using the Breast Imaging Reporting and Data System. For each lesion, the two most experienced radiologists retrospectively reviewed the results together to reach consensus.

Biopsy procedure. CNB or VAB were performed, guided by US or mammography (MMG). If lesions were detected by US, biopsy was performed as a US-guided procedure. If no lesion was detected by US, stereotactic VAB (ST-VAB) was performed. CNB used a 14-G Biopsy System (CR Bard) and VAB used needles of 10 G8- or 11-G Mammotome (Ethicon Endo-Surgery) or 11- or 14-G Vacora (CR Bard). Our standard protocol was to obtain 3-7 core samples per lesion in the US-guided procedure and 5-12 core samples in ST-VAB. In specific cases such as where the amount of tissue obtained was grossly inadequate or targeting difficulty was experienced, the number of samples was increased. On the other hand, when the patient proved uncooperative or declined to continue, or when minor complications such as pain or bleeding were encountered, fewer core samples were obtained. Cases of excisional biopsy were excluded.

Immunohistochemical examination. All specimens were analyzed by pathologists from Tokyo Medical and Dental university, and specimens were considered estrogen receptor (ER)- or progesterone receptor $(\mathrm{PgR})$-positive on immunohistochemistry (IHC) for staining rates $>10 \%$. For HER2 receptor values, IHC 3+ was defined as breast cancer with strong, complete membrane staining observed in at least $10 \%$ of tumor cells. For HER 2 receptor overexpression of $2+$, gene amplification with fluorescence in situ hybridization was not performed in this study.

Data analysis. After review of the postoperative pathologic results, final diagnoses of all lesions were divided into two groups: Pure DCIS, or invasive ductal carcinoma. Medical records were then reviewed. Differences in proportions of categorical data were tested using Fisher's exact probability test. Unless otherwise indicated, significant differences among mean values of numerical data were analyzed using the two-sample two-sided t-test. Predictors of invasive carcinoma underestimation were determined by uni- and multivariate logistic regression analyses. Values of $\mathrm{P}<0.05$ were regarded as statistically significant. All statistical analyses were performed using EZR software (Saitama Medical Center, Jichi Medical University, (http://www. jichi.ac.jp/saitama-sct/SaitamaHP.files/statmed.html), and a graphic user interface for R (The R Foundation for Statistical Computing). More precisely, EZR is a modified version of $\mathrm{R}$ Commander designed to add statistical functions frequently used in biostatistics (6).

\section{Results}

Clinicopathological characteristics. The clinicopathological features of all patients are summarized in Table I. The mean age of all patients was 55 years (range, 33-82 years). A total of 27 patients $(27 \%)$ were finally diagnosed with invasive cancer. In breast examinations, results of palpation were normal in 81 patients $(80 \%)$ and abnormal in 20 patients $(20 \%)$. Mammographic abnormality detected calcification (cal) only in 62 patients (61\%), focal asymmetric density (FAD) only in 8 patients $(8 \%)$, distortion only in 6 patients $(6 \%)$, negative findings in 16 patients $(16 \%)$, and other (FAD+cal, FAD+distortion, or distortion+cal) in 9 patients (9\%).

Enhancement patterns on MRI were categorized as follows: Homogeneous, heterogeneous, clumped, or clustered ring pattern. Enhancement patterns were homogeneous in 1 patient (1\%), heterogeneous in 12 patients (12\%), clumped in 44 patients (43\%), and clustered ring in 32 patients $(32 \%)$.

Median maximum diameter in the 90 patients with measurable lesions on MRI was $46 \mathrm{~mm}$ (range, 10-80 mm). The remaining 11 patients had negative findings for the breast or unmeasurable findings on breast MRI. As $30 \mathrm{~mm}$ was the median, we divided cases into $>30$ or $\leq 30 \mathrm{~mm}$. Twenty-eight patients $(28 \%)$ underwent CNB guided by US, 39 patients (39\%) underwent VAB guided by US, and 34 patients $(34 \%)$ underwent ST-VAB. All patients underwent SLNB, revealing a positive result in only 1 patient. No evidence of metastasis to a non-sentinel lymph node was identified.

Immunohistochemical examination. The frequency of a HER2 score of $0-2+$ was $72 \%$ and that of $3+$ was $20 \%$. HER 2 score 
Table I. Characteristics at the time of biopsy $(n=101)$.

\begin{tabular}{lcc}
\hline Characteristics & $\begin{array}{c}\text { Patients } \\
(\mathrm{n}=101)\end{array}$ & $\begin{array}{c}\text { Percentage } \\
\text { of sample }(\%)\end{array}$ \\
\hline
\end{tabular}

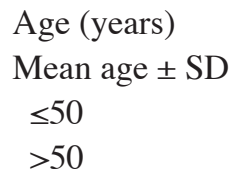

$56.0 \pm 11.2$

$\begin{array}{ll}40 & 40 \\ 61 & 60\end{array}$

Postoperative pathology

DCIS

IDC

Physical examination

Abnormal result of palpation

Normal result of palpation

Mammographic lesion

Calcification

FAD

Distortion

Others

No findings

Enhancement pattern on MRI

Homogenous

Heterogenous

Clumped

Clustered ring

No findings

Maximum lesion size ( $\mathrm{mm}$ )

Mean diameter \pm SD

Range, 10-80 mm

10-29

$30-49$

50-69

$>70$

Other

Biopsy method

US-CNB

US-VAB

ST-VAB

ER

Positive

Negative

Unknown

PgR

Positive
Negative
Unknown
HER2
Positive
Negative
Unknown

Nuclear grade

1,2

3

Unknown
Table I. Continued.

\begin{tabular}{lcc}
\hline Characteristics & $\begin{array}{c}\text { Patients } \\
(\mathrm{n}=101)\end{array}$ & $\begin{array}{c}\text { Percentage } \\
\text { of sample (\%) }\end{array}$ \\
\hline Comedo necrosis & & \\
Absent & 63 & 62 \\
Present & 37 & 37 \\
Unknown & 1 & 1 \\
\hline
\end{tabular}

DCIS, diagnosed ductal carcinoma in situ; IDC, invasive ductal carcinoma; FAD, focal asymmetric density; US, ultrasonography; CNB, core-needle biopsy; VAB, vacuum-assisted biopsy; MRI, magnetic resonance imaging; ER, estrogen receptor; PgR, progesterone receptor; $\mathrm{SD}$, standard deviation.

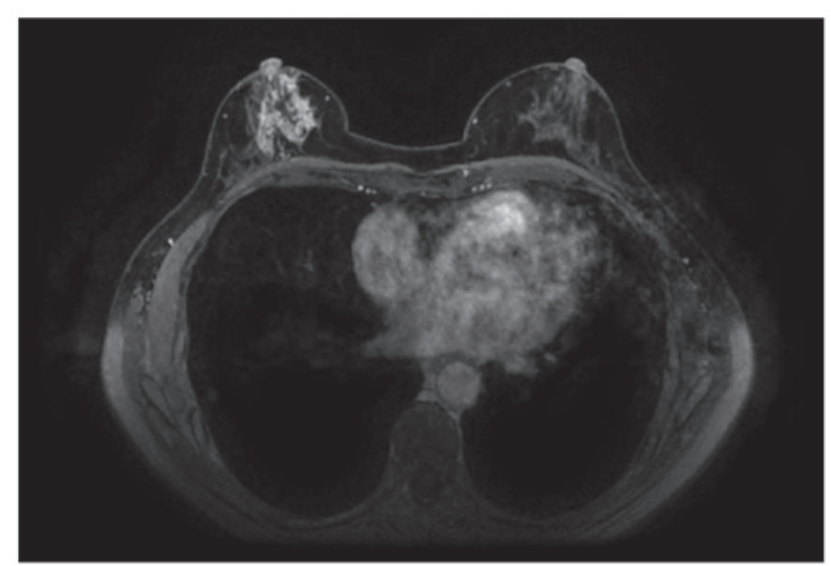

Figure 1. Axial contrast-enhanced T1-weighted fat-suppressed MR subtraction images for DCIS of the right breast demonstrating non-mass-like enhancement. DCIS, diagnosed ductal carcinoma in situ.

was unknown in $8 \%$. ER- and PR-positive statuses were seen in 70 and $64 \%$ of our cases.

ER+/HER2- status was seen in 64\%, ER+/HER2+ status in 9\%, ER-/HER2+ status in 10\%, and ER-/HER2- in 5\%. Among the 19 patients diagnosed with HER2-positive DCIS preoperatively, 9 patients (53\%) were upstaged to IDC.

Predictors of invasive carcinoma underestimation. Univariate analysis of predictors for the presence of invasive components within final specimens initially diagnosed as DCIS is summarized in Table II. The rate of upstaging to invasive cancer in the final pathology was significantly associated with variables such as abnormal results of palpation on breast examination $(\mathrm{P}=0.05)$, comedo necrosis $(\mathrm{P}=0.05)$, and HER2 status $(\mathrm{P}=0.02)$. Multivariate analysis of all factors identified as significant in univariate analyses demonstrated the presence of abnormal palpation as an independent predictor of invasive cancer underestimation (odds ratio 4.76; confidence interval 1.44-15.7; $\mathrm{P}=0.01$ ) (Table III).

\section{Discussion}

To the best of our knowledge, this is the first report to assess predictive factors focusing only on non-mass-type DCIS. 
Table II. Univariate analysis of predictors of invasive cancer in all 101 patients with an initial diagnosis of non-mass DCIS.

Final diagnosis

\begin{tabular}{|c|c|c|c|c|}
\hline Characteristics & $\operatorname{IDC}(n=27)$ & DCIS (n=74) & Underestimation rate (\%) & $P$-value \\
\hline \multicolumn{5}{|l|}{ Age } \\
\hline Mean age $\pm S D$ & $54.3 \pm 10.1$ & $55.4 \pm 11.7$ & & $0.64^{\mathrm{b}}$ \\
\hline Range & $39-71$ & $33-82$ & & \\
\hline \multicolumn{5}{|l|}{ Palpation } \\
\hline Abnormal & 9 & 11 & 45 & \\
\hline Normal & 18 & 63 & 22 & 0.05 \\
\hline \multicolumn{5}{|l|}{ Biopsy method } \\
\hline ST-VAB & 8 & 26 & 23 & \\
\hline US-VAB & 11 & 28 & 28 & \\
\hline US-CNB & 8 & 20 & 28 & 0.88 \\
\hline \multicolumn{5}{|l|}{ MMG } \\
\hline Calcification only or normal & 20 & 58 & 26 & \\
\hline FAD & 2 & 6 & 25 & \\
\hline Distortion & 1 & 5 & 17 & \\
\hline Others & 4 & 5 & 44 & 0.65 \\
\hline $\begin{array}{l}\text { Enhancement pattern on MRI } \\
\text { homogenous }\end{array}$ & 0 & 1 & & \\
\hline Heterogenous & 5 & 7 & & \\
\hline Clumped & 10 & 34 & & \\
\hline Clustered ring & 10 & 22 & & \\
\hline No findings & 1 & 8 & & 0.483 \\
\hline \multicolumn{5}{|l|}{ MRI size (mm) } \\
\hline$<30$ & 12 & 38 & 24 & \\
\hline$\geq 30$ & 14 & 34 & 29 & \\
\hline Unknown & 1 & 2 & & 0.81 \\
\hline \multicolumn{5}{|l|}{ ER } \\
\hline Positive $(\geq 10 \%)$ & 19 & 58 & 25 & \\
\hline Negative & 8 & 14 & 36 & \\
\hline Unknown & 0 & 2 & & 0.48 \\
\hline \multicolumn{5}{|l|}{$\mathrm{PgR}$} \\
\hline Positive $(\geq 10 \%)$ & 18 & 52 & 26 & \\
\hline Negative & 9 & 20 & 31 & \\
\hline Unknown & 0 & 2 & & \\
\hline \multicolumn{5}{|l|}{ HER2 } \\
\hline Positive & 10 & 9 & 53 & \\
\hline Negative & 16 & 59 & 21 & \\
\hline Unknown & 1 & 6 & & 0.02 \\
\hline \multicolumn{5}{|l|}{ Nuclear grade } \\
\hline 3 & 3 & 7 & 30 & \\
\hline 1,2 & 21 & 65 & 24 & \\
\hline Unknown & 3 & 2 & & 0.71 \\
\hline \multicolumn{5}{|l|}{ Comedo necrosis } \\
\hline Present & 14 & 23 & 25 & \\
\hline Absent & 12 & 51 & 19 & \\
\hline Unknown & 1 & 0 & & 0.05 \\
\hline
\end{tabular}

a P-values were estimated using Fisher's exact probability test; ' ${ }^{b}$ Student's t-test. DCIS, diagnosed ductal carcinoma in situ; IDC, invasive ductal carcinoma; FAD, focal asymmetric density; US, ultrasonography; CNB, core-needle biopsy; ST-VAB, stereotactic vacuum-assisted biopsy; MRI, magnetic resonance imaging; ER, estrogen receptor; PgR, progesterone receptor; SD, standard deviation. 
Table III. Multivariate analysis for predictors of invasive cancer in patients with non-mass-type DCIS (n=101).

\begin{tabular}{lccc}
\hline Independent predictors of invasive cancer & Odds ratio & 95\% confidence interval & P-value $^{\mathrm{a}}$ \\
\hline Abnormal palpation & 4.76 & $1.44-15.7$ & 0.01 \\
Comedo necrosis & 2.32 & $0.76-7.14$ & 0.14 \\
CNB HER2 & 2.84 & $0.85-9.46$ & 0.09
\end{tabular}

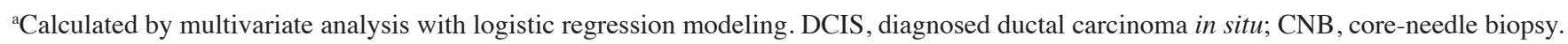

Our study showed that among preoperatively diagnosed non-mass-type DCIS, approximately $27 \%$ were underestimations. In particular, the presence of a clinically abnormal result of palpation appeared to increase the chance of up-staging to invasive cancer.

Many studies have reported preoperative factors that can predict upstaging of DCIS to invasive cancer (2,3,5,7-15). We hypothesized that the factors listed in those reports may be influenced by the forms of DCIS. Factors such as size and existence of a palpable lesion depend on whether the DCIS is mass or non-mass type. For example, among intracystic lesions, the underestimation rate may not be high despite the large, palpable lesion. Our study focused only on non-mass-type DCIS and evaluated preoperative clinicopathological factors predicting underestimation.

The underestimation rate for percutaneous breast biopsy has been reported to range between 15 and $40 \%$ (1-4). In the present study, the underestimation rate was $27 \%$, within the reported range. As biopsy targeted at the component considered invasive is difficult for non-mass-type DCIS, we thought that the underestimation rate may be lower. However, the underestimation rate was unchanged in the present study. The underestimation rate may also change depending on the thickness of the needle, the number of specimens and the number of stereotactic biopsies, thus re-examination of an increased number of cases is necessary.

Findings of this study demonstrated that preoperative factors predictive of the invasive component were abnormal results of palpation, HER2-positive, and comedo necrosis in univariate analysis. Overexpression of HER2 in invasive breast cancer is an independent predictor of poor prognosis. The significance of HER2 overexpression in DCIS is not well defined. However, HER2 DCIS has recently been reported as an aggressive type (16-18). Monabati et al reported that HER2-positive DCIS cases were more likely to be of high nuclear grade (18). Mustafa et al reported that HER2-positive DCIS tended to be upstaged to invasive ductal carcinoma (16). However, the targets of that report were all typed as DCIS. This was the first report to examine HER2 score for non-mass-type DCIS only. The results showed that HER2-positive DCIS tended to be upstaged to invasive cancer, but no significant difference was observed in multivariate analysis. Future studies will accumulate data for these tests from additional cases and further assessment is needed for validation.

In our study, only an abnormal result of palpation was a predictive factor in multivariate analysis. Other studies have reported abnormal palpation as a predictor $(1,15,18)$. On the other hand, Sato et al described abnormal palpation as irrelevant to the presence of invasion (5). However, those reports all examined all-type DCIS, rather than restricting investigation to only non-mass-type DCIS. No previous reports have described abnormal palpation as a predictor in non-mass-type DCIS.

Previous findings have shown that, CNB and thinner needles as significant predictors of underestimation compared to VAB or thicker needles (10). In the present study, different devices were not associated with upstaging. Various factors may have contributed to this finding. One was the difference in the number of cases. Another case involved US guidance in which a lesion with suspected invasiveness was biopsied using $\mathrm{CNB}$ rather than VAB. As a result, the risk of underestimation tended to be reduced with VAB.

The present study has some limitations. First, this study is a single-institution review and retrospective. Nevertheless, the results of the present study may be valuable to other institutions at the time of surgery for cases with a preoperative diagnosis of DCIS. In particular, information containing HER2 status is considered valuable, as our institution routinely tests for the overexpression of HER 2 in all patients with DCIS. Second, the number of cases has been reduced by targeting only non-mass DCIS. As a result, the essential stratification in this study indicated the sample size of each group was reduced. Future validation studies with a large sample set evaluating the risk factors leading to upstaging of DCIS and potential new options in the DCIS treatment algorithm are required.

In conclusion, preoperatively diagnosed non-mass-type DCIS represented an underestimation in approximately $27 \%$ of cases. In particular, the presence of a clinically abnormal result of palpation increases the chances of up-staging to invasive cancer. In cases with an abnormal result from palpation, the surgeon should select the operation in consideration of the possibility of upstaging to IDC. Larger, multi-institutional investigations are necessary to more closely examine risk factors for upstaging of non-mass-type DCIS on breast biopsy to IDC on the final pathology.

\section{Acknowledgements}

Not applicable.

\section{Funding}

No funding was received.

\section{Availability of data and materials}

The datasets used and/or analyzed during the present study are available from the corresponding author on reasonable request. 


\section{Authors' contributions}

GO performed surgery, wrote the manuscript, analyzed the data and performed the statistical analysis. TN, AO, YK, TH, HS and TI performed surgery and collected data. MM, TF and KK were responsible for diagnostic imaging and biopsy. IO was in charge of the pathology. HU designed the present study and wrote the manuscript. The final version was read and adopted by all the authors.

\section{Ethics approval and consent to participate}

The study was approved by the Ethics Committee of Tokyo Medical and Dental University Hospital (Tokyo, Japan). Signed informed consents were obtained from the patients.

\section{Patient consent for publication}

Not applicable.

\section{Competing interests}

The authors declare that they have no competing interests.

\section{References}

1. Lee JW, Han W, Ko E, Cho J, Kim EK, Jung SY, Cho N, Moon WK, Park IA and Noh DY: Sonographic lesion size of ductal carcinoma in situ as a preoperative predictor for the presence of an invasive focus. J Surg Oncol 98: 15-20, 2008.

2. Brennan ME, Turner RM, Ciatto S, Marinovich ML, French JR, Macaskill P and Houssami N: Ductal Carcinoma in situ at core-needle biopsy: Meta-analysis of underestimation and predictors of invasive breast cancer. Radiology 260: 119-128, 2011.

3. Wahedna Y, Evans AJ, Pinder SE, Ellis IO, Blamey RW and Geraghty JG: Mammographic size of ductal carcinoma in situ does not predict the presence of an invasive focus. Eur J Cancer 37: 459-462, 2001.

4. Darling ML, Smith DN, Lester SC, Kaelin C, Selland DL, Denison CM, DiPiro PJ, Rose DI, Rhei E and Meyer JE: Atypical ductal hyperplasia and ductal carcinoma in situ as revealed by large-core needle breast biopsy: Results of surgical excision. AJR Am J Roentgenol 175: 1341-1346, 2000.

5. Sato Y, Kinoshita T, Suzuki J, Jimbo K, Asaga S, Hojo T, Yoshida $\mathrm{M}$ and Tsuda $\mathrm{H}$ : Preoperatively diagnosed ductal carcinoma in situ: Risk prediction of invasion and effects on axillary management. Breast Cancer 23: 761-770, 2016.

6. Kanda Y: Investigation of the freely available easy-to-use software 'EZR' for medical statistics. Bone Marrow Transplant 48: $452-458,2013$

7. Jackman RJ, Burbank F, Parker SH, Evans WP III, Lechner MC, Richardson TR, Smid AA, Borofsky HB, Lee CH, Goldstein HM, et al: Stereotactic breast biopsy of nonpalpable lesions: Determinants of ductal carcinoma in situ underestimation rates. Radiology 218: 497-502, 2001.
8. King TA, Farr GH, Cederbom GJ, Smetherman DH, Bolton JS, Stolier AJ and Fuhrman GM: A mass on breast imaging predicts coexisting invasive carcinoma in patients with a core biopsy diagnosis of ductal carcinoma in situ. Am Surg 67: 907-912, 2001.

9. Hoorntje LE, Schipper ME, Peeters PH, Bellot F, Storm RK and Borel Rinkes IH: The finding of invasive cancer after a preoperative diagnosis of ductal carcinoma-in situ: Causes of ductal carcinoma-in situ underestimates with stereotactic 14-gauge needle biopsy. Ann Surg Oncol 10: 748-753, 2003.

10. Cho N, Moon WK, Cha JH, Kim SM, Kim SJ, Lee SH, Chung HK, Cho KS, Park IA and Noh DY: Sonographically guided core biopsy of the breast: Comparison of 14-gauge automated gun and 11-gauge directional vacuum-assisted biopsy methods. Korean J Radiol 6: 102-109, 2005.

11. Yen TW, Hunt KK, Ross MI, Mirza NQ, Babiera GV, Meric-Bernstam F, Singletary SE, Symmans WF, Giordano SH, Feig BW, et al: Predictors of invasive breast cancer in patients with an initial diagnosis of ductal carcinoma in situ: A guide to selective use of sentinel lymph node biopsy in management of ductal carcinoma in situ. J Am Coll Surg 200: 516-526, 2005.

12. Cox D, Bradley S and England D: The significance of mammotome core biopsy specimens without radiographically identifiable microcalcification and their influence on surgical management-a retrospective review with histological correlation. Breast 15: 210-218, 2006.

13. Dillon MF, McDermott EW, Quinn CM, O'Doherty A, O'Higgins $\mathrm{N}$ and Hill AD: Predictors of invasive disease in breast cancer when core biopsy demonstrates DCIS only. J Surg Oncol 93: 559-563, 2006.

14. Rutstein LA, Johnson RR, Poller WR, Dabbs D, Groblewski J, Rakitt T, Tsung A, Kirchner T, Sumkin J, Keenan D, et al: Predictors of residual invasive disease after core needle biopsy diagnosis of ductal carcinoma in situ. Breast J 13: 251-257, 2007.

15. Trentin C, Dominelli V, Maisonneuve P, Menna S, Bazolli B, Luini A and Cassano E: Predictors of invasive breast cancer and lymph node involvement in ductal carcinoma in situ initially diagnosed by vacuum-assisted breast biopsy: Experience of 733 cases. Breast 21: 635-640, 2012.

16. Mustafa RE, DeStefano LM, Bahng J, Yoon-Flannery K, Fisher CS, Zhang PJ, Tchou J, Czerniecki BJ and De La Cruz LM: Evaluating the risk of upstaging HER2-positive DCIS to invasive breast cancer. Ann Surg Oncol 24: 2999-3003, 2017.

17. Di Cesare P, Pavesi L, Villani L, Battaglia A, Da Prada GA, Riccardi A and Frascaroli M: The relationships between HER2 overexpression and DCIS characteristics. Breast J 23: 307-314, 2017.

18. Monabati A, Sokouti AR, Noori SN, Safaei A, Talei AR, Omidvari S and Azarpira N: Large palpable ductal carcinoma in situ is Her-2 positive with high nuclear grade. Int J Clin Exp Pathol 8: 3963-3970, 2015. 